\title{
Final design of the JT-60SA pellet launching system for simultaneous density and ELM control
}

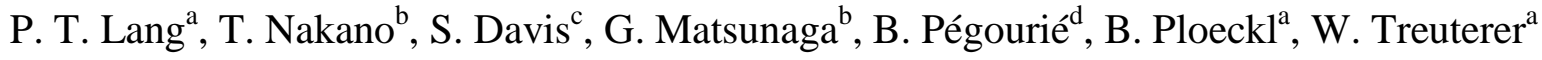 \\ ${ }^{a}$ Max Planck Institute for Plasma Physics, EURATOM Association, Boltzmannstr. 2, 85748 Garching, Germany \\ ${ }^{b}$ National Institutes for Quantum and Radiological Technology, Naka Fusion Institute, 801-1 Mukoyama, Naka-shi, \\ Ibaraki-ken, Japan 311-0193 \\ ${ }^{c}$ Fusion for Energy, EURATOM Association, Boltzmannstr. 2, 85748 Garching, Germany \\ ${ }^{d}$ CEA, IRFM, 13108 Saint-Paul-lez-Durance Cedex, France
}

\begin{abstract}
The key mission of the new tokamak JT-60SA is to conduct exploitations in view of ITER and to resolve key physics and engineering issues for DEMO. Its pellet launching system was designed to cover according requirements by providing a powerful and flexible tool for the control of density profile and ELM frequency. Modelling showed inboard launch is mandatory in order to achieve high fuelling efficiency; by analysing the potential pellet fuelling impact in all relevant plasma scenarios the optimized set of pellet parameters for fulfilling all the tasks requirements was elaborated. Accordingly, the systems layout had to be adapted for pellet injection via a guiding tube from the vessel inboard side. Currently, efforts are under way in order to elaborate the most appropriate solution yielding minimised pellet transfer losses and a maximum transfer speed yet compatible with boundary conditions imposed by the vessel already under construction. The feasibility of a mechanical centrifuge as pellet acceleration unit was studied. This approach would guarantee the precise pellet launch speed as needed to enable the best adaptation to the guiding tube transfer capabilities and accurate control of pellet frequency and particle flux as arriving in the plasma. While the appropriateness of the centrifuge principle has been already proven by several devices, the proposed version possesses a novel design employing several accelerator arms. Such, operation at heighten rates and with a more refined adjustment of pellet flux and frequency could be achieved. Moreover, it allows hosting several sources delivering different pellets and their simultaneous actuation. The appropriate control unit is designed to merge pellets from different sources to form a sequence for the simultaneous control of different basic plasma parameters as e.g. density and ELM frequency. This flexibility can also allow including additional applications, like isotope fraction control or radiative power exhaust by using compound pellets. At present, the detailed engineering of all major components is in progress, finally aiming to provide specifications and get ready to prepare the procurement process.
\end{abstract}

Keywords: Tokamak, Pellet fuelling, Launcher technology, JT-60SA

\section{Introduction}

The task of this ongoing project is to provide a pellet launching system (PLS) for the new device JT-60SA in due time for the initial research phase II currently planned to start in early 2023. JT-60SA [1] is a tokamak using superconducting toroidal and poloidal field coils with a mission to contribute to early realization of fusion energy by supporting the exploitation of ITER and by resolving key physics and engineering issues for DEMO reactors. There are two main tasks assigned to the PLS: particle fuelling and ELM pacing. Particle fuelling has to enable access to the high-density regime beyond the empirical Greenwald limit; technically a feedback control of the density gradient has to be facilitated. For the envisaged role of controlling the ELM activity, the physics task is to sound out the pellet potential for ELM triggering. In case pellets are found capable to initiate ELMs, the technical task is to optimize pellet parameters in order to minimize the fuelling impact and provide a pacing tool for the feedback control of the ELM frequency potentially applied for ELM mitigation.

Initial investigation took into account that the torus vacuum vessel is already under construction and hence any approach must comply with the existing boundary conditions. It turned out that there are only few options remaining for pellet injection into the plasma. Modelling the fuelling impact of single pellets, adopting the technical boundary conditions of every technically possible solution unveiled that pellet launch from the torus inboard side is the best approach for any of the considered plasma operational scenarios [2]. This is despite the finally chosen inboard launch option requires pellet transfer through a multi-bend guiding system. For the prescribed pellet size range, this restricts the employable pellet speed to the range $200-500 \mathrm{~m} / \mathrm{s}$.

For the pacing task, required pellet sizes are most likely much smaller than those of fuelling pellets. To ensure reliable delivery, however, a minimum residual size of about $10 \%$ of the fuelling pellet mass is necessary and envisaged. Foreseen pellet pacing rates are likely to exceed the injection rate of the fuelling pellets by a factor of up to $5-10$. In turn, for scenarios where pacing pellets cause sufficient impact to trigger an ELM fuelling pellets for sure will do so as well. It is thus obvious there will be a strong interference between fuelling and pacing actuation. However, by controlling both parameters simultaneously it is possible to take advantage of this "cross-talk". The system layout and the suggested approach for the launch control are made accordingly 
Finally, experience with existing pellet systems has shown some headroom in the operational features beyond the initially prescribed scope, which can be of great benefit. Consequently, the proposed design comprises several options for upgrades. These would enable the system to cover additional points of interest such as the efficient handling of plasma enhancement gases or isotopic mixtures for burn control simulation.

\section{System design}

\subsection{Adaptation to the boundary conditions}

As already noted previously, the major boundary condition to be coped with is the fact that JT-60SA's vacuum vessel is already under construction and therefore guiding tubes required for pellet transfer have been subject to significant constructional restrictions. Currently, several options to route the guiding tube, enabling pellet inboard launch, are being investigated. Possible solutions are evaluated employing a simple heuristic approach. First, any considered route is approximated by a series of circular sections characterised by their curvature radii $\mathrm{R}_{\mathrm{i}}$. Then, for this series an effective bend radius $\mathrm{R}_{\mathrm{eff}}$ for the approximated route is estimated by

$$
R_{\text {eff }}=1 / \sqrt{\sum_{i} \frac{1}{R_{i}^{2}}} .
$$

Finally, the critical transfer velocity $\mathrm{v}_{\mathrm{c}}$ is calculated for $\mathrm{a}$ pellet with length $L$ (to be expressed in the units of $R_{e f f}$ ) by applying the empirical AUG calibrated relation [2]

$v_{c}=36.4\left[\frac{m}{s}\right] \sqrt{\frac{R_{e f f}}{L}}$.

Notably, the AUG calibration factor was derived for varied L values but cubic D pellets only. Differing pellet shapes, ice mixtures and ice processing will most likely affect it. Yet, for an initial estimation and design optimisation the approach is considered sufficiently reasonable. Design optimisation is seeking to maximize $\mathrm{v}_{\mathrm{c}}$ and hence $\mathrm{R}_{\mathrm{eff}}$. Reasoning for this is that the pellet speed is found to be advantageous for the performance of pellet fuelling in the sense that fast pellets can achieve the same density increase with less particle flux. Due to the necessity of inserting a multi bend guiding tube the maximum possible pellet transfer speed will be considerably constricted; estimations yield a critical transfer speed of $470 \mathrm{~m} / \mathrm{s}$ for the envisaged fuelling pellets. Consequently, the acceleration system had to be adapted in order to deliver pellets in the accessible transfer speed range. A precise speed control and a small speed scatter are needed in order to achieve a perfect match to the optimum transfer speed. Beyond its need for adaptation purposed, the precise control of the pellet launching speed at the exit of the acceleration system is also necessary for the requested control performance. Due to a significant distance between accelerator exit and plasma entrance position, significant speed scatter would result in significant flight-time scatter causing distortions of pellet arrival times and hence of the applied frequency. Mainly for these reasons, a centrifuge accelerator was chosen. This type of acceleration units have already proven to yield a high precision in pellet speed control, in combination with very low speed scatter while providing reliable pellet delivery [3]. Furthermore, our design takes advantage of the centrifuge features enabling it to establish pellet trains combined from both fuelling and pacing pellets. Equipped with a corresponding controlling unit, it thus will be able to provide an integrated and smart solution for simultaneous actuation on fuelling and pacing. It is capable to take into account the fuelling impact of pacing pellets and the pacing impact of fuelling pellets. It is understood that the pellet sources have to be designed to cope with the arising boundary conditions. And, for the pellet sources as well, a design has been derived covering all basic needs. The proposed design is also rendering the possibility of an upgrade, in order to allow for more sophisticated applications.

\subsection{Layout of the major components}

\section{Pellet sources}

Separate sources are needed for the fuelling and the pacing task, due to the expected significantly different masses. Since JT-60SA envisages steady state operation, any source has to be capable to deliver sufficiently long pellet trains. Consequently, continuously working screw extruders have to be employed. For this type of pellet production units, delivery of mass fluxes, pellet sizes and rates approaching reactor grade needs has been already demonstrated [4]. In addition, to make ice with a consistency well suitable for pellet production, the handling of any required hydrogen isotope is possible. Since operation with deuterium (D) will have highest priority, the layout is optimised for this isotope while operation with protium $(\mathrm{H})$ is kept as an option.

For the fuelling extruder, according to the request expressed in the research plan and confirmed by detailed modelling, a pellet mass of $\mathrm{m}_{\mathrm{P}}=6.5 \times 10^{20} \mathrm{D}$ atoms is envisaged. This is due to cylindrical pellets with diameter $\varnothing$ and length $\mathrm{L}$ each $2.4 \mathrm{~mm}$. In order to provide some flexibility but still keeping a robust pellet shape, adjustment of $\mathrm{L}$ within the range $\varnothing / 2$ to $2 \varnothing$ is foreseen, allowing varying $\mathrm{m}_{\mathrm{P}}$ by a factor of 4 . The maximum extruder throughput is set to $\Gamma=1.3 \times 10^{22} \mathrm{D} / \mathrm{s}$, corresponding to a pellet rate of $f_{P}=20 \mathrm{~Hz}$ for the reference $\varnothing=\mathrm{L}$ pellets.

The major request for pellet pacing refers to the repetition rate; in order to provide a tool for ELM control, at a sufficiently high frequency here $f_{P}=50 \mathrm{~Hz}$ is asked for. For pacing purposes, in order to minimize the fuelling side effect, a pellet size as small as possible is preferred. Yet, reliable pellet delivery to the plasma has to be granted. A pellet size of $\varnothing=\mathrm{L}=1.2 \mathrm{~mm}$ was chosen, which is regarded to be sufficient for yielding reliable transfer through the entire guiding system. Again, mass adaption to actual requirements is foreseen. In addition to a fast response via $\mathrm{L}$ variation, here the exchange of the extrusion nozzle to alter $\varnothing$ is foreseen. Notably, in this start-up configuration, pellet ELM pacing at $50 \mathrm{~Hz}$ would cause a particle flux of about 
$\Gamma=0.4 \times 10^{22} \mathrm{D} / \mathrm{s}$, unlikely to be negligible when simultaneously density control is requested.

Centrifuge pellet acceleration unit

As pellet acceleration unit, a stop cylinder centrifuge known for its capability of precise speed and frequency control [3] will be employed. In order to allow simultaneous pellet delivery by several extruders, a stop cylinder radius $\mathrm{r}_{0}$ of $0.4 \mathrm{~m}$ is foreseen. Thus, up to 6 pellet sources could be equipped to a single centrifuge unit. To ideally adapt the launch speed of this centrifuge to the accessible transfer speed range, a straight double acceleration arm with a radius $\mathrm{R}$ of $0.23 \mathrm{~m}$ has been chosen. Yielding a relation of about $\mathrm{v}_{\mathrm{P}}=2[\mathrm{~m}] \mathrm{x} \mathrm{f}_{\mathrm{C}}$ between pellet speed and centrifuge revolution frequency $\mathrm{f}_{\mathrm{C}}$, this optimizes the accessible $\mathrm{f}_{\mathrm{P}}$ range as well. Employing the double arm, thus a maximum $\mathrm{f}_{\mathrm{P}}=500 \mathrm{~Hz}$ is possible, potentially enabling the access to pacing rates up to $200-300 \mathrm{~Hz}$ in case. Possibly, some advanced plasma scenarios that call for higher pellet flux could be covered by extending the system by adding further fuelling size pellet extruders. And finally, the option of running several pellet sources in parallel provides the amenity to add a device processing an alternative pellet composition, such as hydrogen isotope mixtures or admixture of other gases.

With the chosen layout, a fine tuning of the pellet rate is possible. Due to the acceleration principle and since usually the centrifuge revolution frequency $f_{C}$ is kept constant, only discrete values for the pellet rate can be realised. For the double arm layout, rates have to satisfy the relation $f_{P}=2 f_{C} / n$ with $n$ integer. Since the envisaged pellet speed of $500 \mathrm{~m} / \mathrm{s}$ is significantly lower than the available technical potential of centrifuge accelerators [5], it can be expected engineering demands to the JT-60SA acceleration unit can remain quite relaxed. To note, since acceleration is mass independent, fuelling and pacing pellets will achieve identical speed. Consequently, a pellet train launched can be composed by any arbitrary sequence of fuelling and pacing pellets. Pellet guiding system

For the chosen solution of the inboard launch, it is necessary to transfer the pellets through guiding tubes. The possible layout for this route is constrained by the need to insert it into an almost fully fixed vessel design. Details and resulting pellet speed restrictions have been already discussed and estimated in [2]; currently efforts are under way to find the optimum possible routing. According to the actual planning, the installation of the in-vessel guiding tubes will take place during the first maintenance and enhancement phase. Yet, the final route and hence the according maximum pellet transfer speed needs to be found. In any case, a fine tuning of the pellet speed to the finally required value is possible with the proposed centrifuge accelerator.

\subsection{System layout overview}

Combined and interacting together, all the major components form a pellet launching system that is fully capable to serve both for fuelling and pacing requests as formulated in the research plan. An overview of the entire system is presented in tables I and II, distinguishing between the basic start up configuration shown in table I and the possible maximum extension displayed in table II. The approach is based on the installation of a single centrifuge acceleration unit. In case a further unit is installed, potentially possible due to the two available inboard guiding tubes, even higher fuelling fluxes and ELM pacing rates up to about $500 \mathrm{~Hz}$ would become possible. A schematic illustration of the system design is shown in figure 1, indicating as well the projected responsibilities for all major components respectively the different sections of the PLS.

\begin{tabular}{|l|}
\hline Pellet sources : \\
2 steady state extruders \\
Operation in $\mathrm{D}$, alternatively in $\mathrm{H}$ \\
Fuelling: \\
$\varnothing=2.4 \mathrm{~mm}, \mathrm{l}=1.2-4.8 \mathrm{~mm}$ \\
$\mathrm{~m}_{\mathrm{P}}=3.3-13.0 \times 10^{20}$ atoms (for $\mathrm{D}$ ) \\
$\mathrm{f}_{\mathrm{P}}$ : up to $20 \mathrm{~Hz}$ (at $\left.\mathrm{l}=2.4 \mathrm{~mm}\right)$ \\
$\Gamma_{\mathrm{P}}$ : up to $1.3 \times 10^{22} / \mathrm{s}$ (for $\left.\mathrm{D}\right)$ \\
Pacing: \\
$\varnothing=\mathrm{l}=1.2 \mathrm{~mm}$ \\
$\mathrm{~m}_{\mathrm{P}}=0.8 \times 10^{20}$ atoms (for $\left.\mathrm{D}\right)$ \\
$\mathrm{f}_{\mathrm{P}}:$ up to $50 \mathrm{~Hz}$ \\
\hline Pellet acceleration: \\
Stop cylinder double arm centrifuge \\
$\mathrm{f}_{\mathrm{C}}=100-250 \mathrm{~Hz}$ \\
$\mathrm{~V}_{\mathrm{P}}=200-500 \mathrm{~m} / \mathrm{s}$ \\
$\mathrm{f}_{\mathrm{P}}$ : up to $2 \mathrm{x} \mathrm{f}_{\mathrm{C}}=200-500 \mathrm{~Hz}$ \\
\hline Pellet guiding: \\
Guiding tubes - two variants \\
Inboard (tilted by $70^{\circ}$ ) \\
\hline
\end{tabular}

Table I: Main parameters of the itemized JT-60SA pellet system order for the envisaged start-up configuration.

\section{Pellet sources :}

Up to 6 sources

Any combination of fuelling, pacing and test extruders or batch sources

Fuelling:

$\Gamma_{\mathrm{P}}$ : up to $5 \times 10^{22} / \mathrm{s}$ (for D)

Pacing:

Exchangeable nozzel with different $\varnothing$

$\mathrm{f}_{\mathrm{p}}$ : up to $250 \mathrm{~Hz}$

Test:

Operation with HD mixtures or with ice doped with gases for plasma enhancement or radiative power exhaust

Table II: Possible upgrade making full use of the source bearing capacity. Parameters for acceleration and transfer units remain.

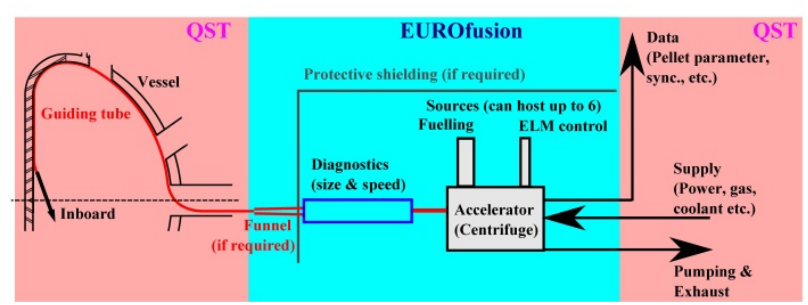

Figure 1: JT60-SA PLS scheme with projected responsibility as shared between QST and EUROfusion. 


\section{Combined fuelling and pacing control}

Any feedback control approach employing a centrifuge acceleration unit must take into account the according specific features of this device. There is on the one hand the fact only a discrete spectrum of $f_{P}$ values is possible and hence the resulting particle fluxes, $\Gamma_{\mathrm{P}}=\mathrm{m}_{\mathrm{P}} \times \mathrm{f}_{\mathrm{P}}$, also only can take on discrete values. On the other hand, already at the moment a pellet request is made a precise prediction of the expected pellet arrival time on plasma is possible. Thus, the control system can initiate appropriate actuation already before the pellet impact takes place; this response time is typically in the order of $100 \mathrm{~ms}$ ! Typical related actions are e.g. the notching of wave heating to avoid strong reflections from the ablation region or corrective actions in measurements of key plasma parameters disturbed by the pellet impact.

Algorithms mastering the discrete nature of the pellet particle flux, while taking advantage of the precise prediction of pellet arrival have been developed and successfully applied in the tokamak experiment ASDEX Upgrade [6]. In these experiments, the algorithm was used to control fuelling by applying a PLS with a centrifuge employing just a single acceleration arm and equipped with only a single pellet source. This existing scheme has now to be extended to control two acceleration arms and several pellet sources. Therefore, obviously the JT-60SA centrifuge has to be equipped with at least two pellet sources; in case of combined fuelling and pacing control with each of the two types.

A sketch of the envisaged control scheme is shown in figure 2; the example displayed incorporates 4 different pellet sources. Their type is not specified. However, any type could represent a source for fuelling or pacing pellets - or a source delivering pellets for another task like e.g. mixture pellets for radiative power exhaust control [7]. For every source, pellet material and size are preselected before the pellet tool can be applied within a plasma discharge. These parameters have to be communicated (prior to the experiment) to the plasma control system, together with the source characteristic like e.g. the maximum available repetition rate. Every time the revolving centrifuge is passing the reference position $\left(\varphi \equiv 0^{\circ}\right)$, a synchronization signal is created and sent to the controller. This signal indicates the actual position of both arms and enables the control system to calculate the actual pellet speed and the expected times of pellet arrival in the plasma.

In this configuration, two pellets can be launched per revolution cycle. Both "slots" can be attributed to their related arm (A or B). Definitely, only a single pellet can be filled into a slot. Hence, a pellet delivered from an extruder placed early in the centrifuge revolution cycle (starting when the arm passes the exit of the stop cylinder) blocks this slot for the entire remaining cycle. Consequently, extruders placed earlier in the cycle can preclude actuation of extruders positioned later. Thus, these early positions should be filled by sources with the highest priority. Finally, filling of the available slots has to be mastered by the control algorithm taking into account all the actual control requests. For this, eventually differing requirements have to be prioritised taking into account inhibit times needed for the recharging of a source (since the maximum source rate is usually much smaller than $f_{C}$ ). As for any slot, the arrival time on plasma can be calculated, and anticipatory controlling can be achieved. This includes the accounting of pacing pellets for fuelling and vice versa of fuelling pellets for pacing. It is assumed that the highest priority will be assigned to the fuelling source(s) since every fuelling pellet facilitates the full potential of a pacing pellet while the reverse does not hold.

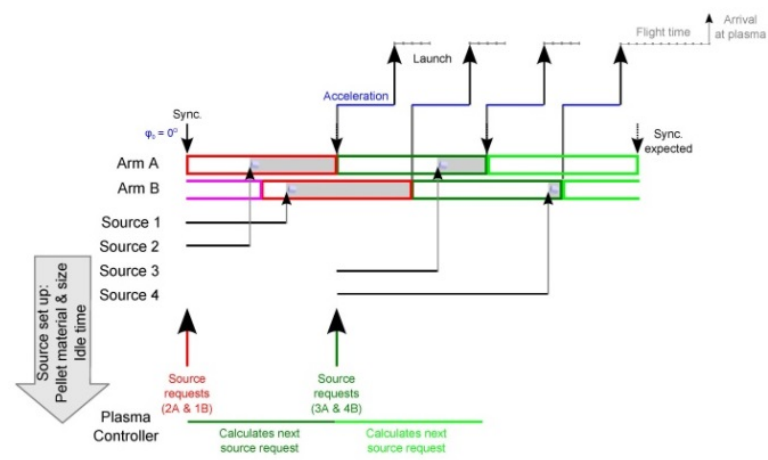

Figure 2: Control scheme sketch for double arm centrifuge, here equipped with four different pellet sources. Per revolution, two "launching slots" can be filled with a single pellet. Provided by all relevant pellet data including a sync clock, the controller can occupy these slots as requested by the various control requirements and precisely predict pellet arrival in the plasma.

\section{Project plan for the manufacturing}

With the conceptual design finished in time, now preparations are ongoing in order to work out a detailed design and initiate the manufacturing of all major components. A project plan, shown in figure 3, has been worked out for a 3 years term, starting with approval after the final conceptual review and aiming to provide finally a pellet launching system that is ready for shipping to JT-60SA. It covers the development and testing of all required major components, with the exception of the major part of the transfer system, the guiding tubes installed inside the torus vessel. Foreseen is the initial installation of two steady-state extruders, one dedicated for fuelling and one for pacing. Both will be equipped with the corresponding gas supply, cooling and vacuum system. At present, it is assumed that both extruders will employ the proven twin-screw technology developed at ORNL [4].

Yet, it is envisaged to build a single centrifuge accelerator, embedded in his local vacuum system and equipped with all the required control capacity. At present, the decision is still pending if the centrifuge will be built relying on either well proven but already somewhat outdated technology [5] or following a proposal [8] employing a novel approach expected to yield more operational flexibility. To allow for a proper testing and pre-commissioning of the entire system, the required diagnostics equipment for pellet quality and performance analysis will be supplied as well. And finally, the connection of the launching system to the guiding tubes will be tested. Here, eventually a 
funnelling section has to be provided in order to bridge a potential gap.

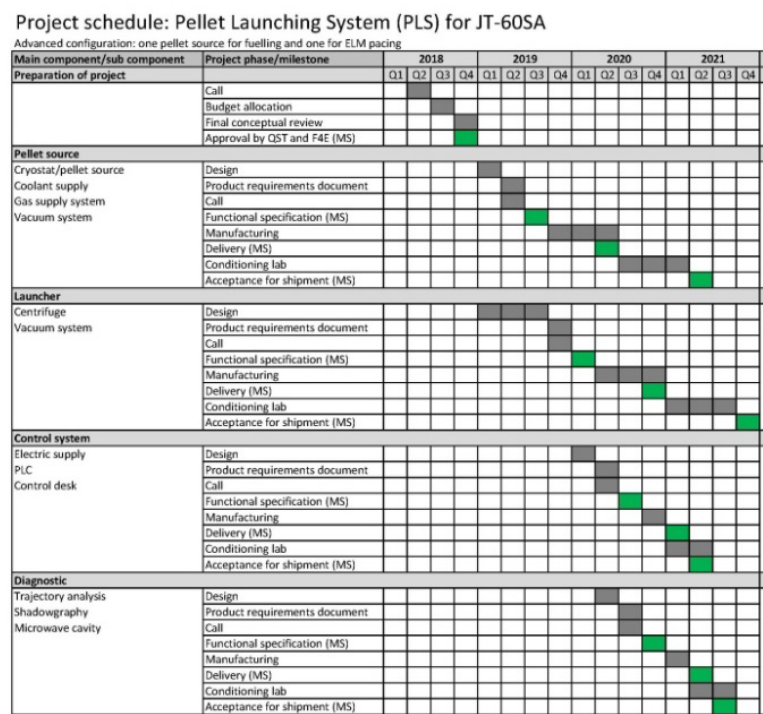

Figure 3: Project schedule proposed for the development of the pellet launching system excluding the in vessel transfer system. Due to this plan, the system would be available for shipping at the end of a 3 years term, starting with approval after the final conceptual review.

\section{Acknowledgments}

This work has been carried out within the framework of the EUROfusion Consortium and has received funding from the Euratom research and training programme 2014-2018 under grant agreement No 633053. The views and opinions expressed herein do not necessarily reflect those of the European Commission.

\section{References}

[1] JT-60SA Research Unit, JT-60SA Research Plan "Research

Objectives and Strategy”, Version 4.0, September 2018 http://www.jt60sa.org/pdfs/JT-60SA_Res_Plan.pdf

[2] P.T. Lang et al., Fusion Eng. Design, 123 (2017) 167

[3] B. Ploeckl et al., Fusion Eng. Design, 86 (2011), 1022

[4] S.J. Meitner et al. Fusion Sci. and Tech. 56 (2009) 52

[5] C. Andelfinger et al., Rev. Sci. Instr., 64, (1993) 983

[6] P.T. Lang et al., Nuclear Fusion, 58 (2018) 036001

[7] A. Kallenbach et al., Nuclear Fusion, 57 (2017) 102015

[8] B. Ploeckl et al., P2.005, this conference 